\title{
Potencial energético dos resíduos do processamento de café combinados com finos de carvão vegetal
}

\author{
Energy potential of coffee processing residues combined with charcoal fines \\ Potencial energético de los residuos del procesamiento del café combinados con los finos del \\ carbón vegetal
}

\section{Resumo}

O objetivo do presente trabalho foi avaliar a composição e a qualidade dos resíduos do processamento de café e suas misturas com finos do carvão vegetal para fins energéticos. A biomassa do café, os finos de carvão vegetal e as misturas $(10,20$ e $30 \%$ de finos de carvão vegetal) foram caracterizados quanto à umidade, composição química imediata e elementar, poder calorífico superior e líquido, e densidade a granel e energética. A inclusão de finos de carvão vegetal promoveu a redução significativa da umidade da biomassa lignocelulósica de café, assim como, o aumento das propriedades energéticas. O tratamento com $30 \%$ de finos de carvão vegetal apresentou a melhor qualidade, atingindo um poder calorífico superior de $19,44 \mathrm{MJ} . \mathrm{Kg}^{-1}$ e poder calorífico líquido de $16,75 \mathrm{MJ}_{\mathrm{Kg}} \mathrm{Kg}^{-1}$, ou seja, um aumento energético de 6 e $10 \%$, respectivamente, em relação ao tratamento com $100 \%$ de biomassa residual de café. As características físico-químicas tanto dos resíduos gerados durante o processamento do café quanto dos finos de carvão apresentam boas propriedades energéticas, que as transformam em um excelente material para utilização no desenvolvimento de biocombustíveis. Portanto, recomenda-se a mistura de pelo menos $20 \%$ de finos de carvão vegetal para potencializar o desempenho energético de resíduos oriundos da produção de café.

Palavras-chave: Resíduo agrícola; Biomassa lignocelulósica; Biocombustíveis.

\begin{abstract}
The objective of the present work was to evaluate the composition and quality of coffee processing residues and their mixtures with charcoal fines for energy purposes. The coffee biomass, charcoal fines and mixtures $(10,20$ and $30 \%$ of charcoal fines) were characterized for moisture, immediate and elemental chemical composition, gross and liquid heating value, and bulk and energy density. The inclusion of charcoal fines promoted a significant reduction in the moisture content of the coffee lignocellulosic biomass, as well as an increase in the energy properties. The treatment with $30 \%$ charcoal fines presented the best quality, reaching a higher calorific value of $19.44 \mathrm{MJ}_{\mathrm{Kg}} \mathrm{Kg}^{-1}$ and
\end{abstract}


liquid heating value of $16.75 \mathrm{MJ} . \mathrm{Kg}^{-1}$, that is, an energy increase of 6 and $10 \%$, respectively, compared to the treatment with $100 \%$ coffee waste biomass. The physicochemical characteristics of both the residues generated during coffee processing and the coal fines show good energetic properties, which make them an excellent material for use in the development of biofuels. Therefore, it is recommended to mix at least $20 \%$ of charcoal fines to enhance the energy performance of residues from coffee production.

Keywords: Agricultural residue; Lignocellulosic biomass; Biofuels.

\section{Resumen}

El objetivo del presente trabajo fue evaluar la composición y calidad de los residuos del procesamiento del café y sus mezclas con finos de carbón vegetal para fines energéticos. La biomasa de café, los finos de carbón vegetal y las mezclas $(10,20$ y $30 \%$ de finos de carbón vegetal) se caracterizaron en cuanto a humedad, composición química inmediata y elemental, poder calorífico bruto y líquido, y densidad aparente y energética. La inclusión de finos de carbón vegetal promovió una reducción significativa del contenido de humedad de la biomasa lignocelulósica de café, así como un aumento de las propiedades energéticas. El tratamiento con $30 \%$ de finos de carbón vegetal presentó la mejor calidad, alcanzando un mayor poder calorífico de $19,44 \mathrm{MJ} \cdot \mathrm{Kg}^{-1}$ y un poder calorífico líquido de

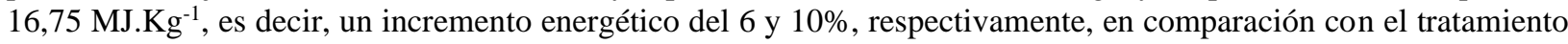
con $100 \%$ de biomasa de residuos de café. Las características fisicoquímicas tanto de los residuos generados durante el procesamiento del café como de los finos de carbón muestran buenas propiedades energéticas, lo que los convierte en un excelente material para su uso en el desarrollo de biocombustibles. Por lo tanto, se recomienda mezclar al menos un $20 \%$ de finos de carbón para mejorar el rendimiento energético de los residuos de la producción de café.

Palabras clave: Residuos agrícolas; Biomasa lignocelulósica; Biocombustibles.

\section{Introdução}

A utilização de resíduos lignocelulósicos, principalmente os oriundos de atividades agroindustriais, tem ajudado a diversificar a matriz energética e reduzir a dependência de recursos petroquímicos. Ao mesmo tempo, é uma alternativa sustentável de gestão de resíduos, além de melhorar o valor agregado do material (Okolie et al., 2021; Solarte-Toro et al., 2018; Souza et al., 2020). O interesse mundial em reduzir os impactos ambientais causados pela atividade industrial é um dos motivos do aumento das pesquisas envolvendo biomassas lignocelulósicas visando diferentes utilizações para agregar valor. A biomassa agroindustrial lignocelulósica é um material de baixo valor agregado, renovável, abundante e com boa relação custo-benefício para geração de bioenergia (Anwar et al., 2014).

As biomassas vegetais são compostas principalmente por celulose, hemicelulose e lignina que estão associados uns aos outros em diferentes graus e composição, dependendo do tipo e da origem da biomassa (Bajpai, 2016; Tian et al., 2018). Ainda que a lignina apresente o maior poder calorífico, as holoceluloses são as responsáveis por gerar uma maior quantidade de energia, já que estão presentes em maior quantidade na biomassa (Demirbas, 2002).

A produção de café gera uma enorme quantidade de resíduos sólidos, podendo atingir a marca de 1,1 toneladas de resíduos para cada tonelada de grão de café processado (International Coffee Organization, 2021; Angeloni et al., 2019). Já que o café é uma das principais commodities do mundo, a tendência de consumo mundial é aumentar cerca de 1,9\% somente na safra de 2021 em comparação com a do ano anterior, a geração de resíduos oriundos do processamento do grão também deve aumentar.

O Brasil é o maior produtor de carvão vegetal do mundo, considerando o ano de 2018, a produção foi de aproximadamente 5,7 milhões de toneladas. Durante a produção do carvão vegetal, é gerado uma grande de finos de carvão que são considerados resíduos devido a sua baixa granulometria e elevada friabilidade. Como a geração de finos pode chegar a cerca de $25 \%$ do total produzido, podemos estimar que somente no ano de 2018, cerca de 1,4 milhão de toneladas de finos foram geradas (Awan et al., 2020; Dias Júnior et al., 2021). Diante do elevado volume gerado é importante desenvolver formas de aproveitamento ecologicamente responsável destes resíduos. Desta forma, o objetivo do presente estudo foi avaliar a composição e a qualidade de resíduos de processamento de café e suas misturas com finos do carvão vegetal para fins energéticos. 


\section{Material e Métodos}

\subsection{Amostragem}

Para a realização do estudo foram utilizados os resíduos de finos de carvão vegetal e do processamento dos grãos de café, assim como, suas misturas (Figura 1). Os finos de carvão vegetal foram obtidos a partir da carbonização de madeiras do fuste e dos galhos de Eucalyptus spp. Os resíduos provenientes do processamento dos grãos de café (Coffea arabica L.) foram coletados na cidade de Machado, Minas Gerais, Brasil.

Figura 1. Resíduos utilizados no estudo. (A) Finos de carvão vegetal; (B) Resíduo do processamento do café; (C) Misturas dos resíduos A x B.
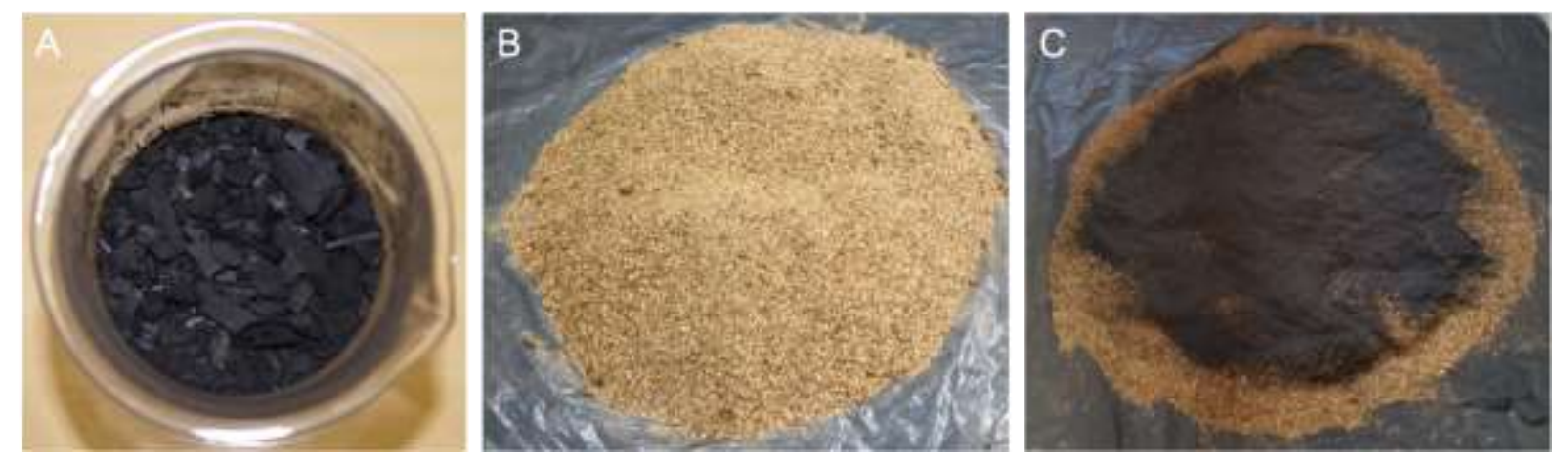

Fonte: Autores.

\subsection{Caracterização dos resíduos}

Os resíduos foram acondicionados para a secagem em condições de umidade de aproximadamente 15\% e classificados quanto ao tamanho das partículas. As frações inferiores a $6 \mathrm{~mm}$ foram utilizadas na composição das misturas realizadas (Tabela 1).

Tabela 1. Composição das misturas realizadas.

\begin{tabular}{cc} 
Tratamentos & Composição (base seca) \\
\hline T1 & 100\% de cascas de café \\
T2 & $90 \%$ de cascas de café $+10 \%$ de finos de carvão vegetal \\
T3 & $80 \%$ de cascas de café $+20 \%$ de finos de carvão vegetal \\
T4 & $70 \%$ de cascas de café $+30 \%$ de finos de carvão vegetal
\end{tabular}

Fonte: Autores.

A umidade base seca e úmida, tanto dos finos de carvão quanto das misturas realizadas, foram determinadas de acordo com as diretrizes da norma DIN EN 14774-1 (Deutsches institut für normung, 2010). A densidade a granel foi calculada pela razão entre a massa e o volume da amostra, utilizando uma proveta de $100 \mathrm{~mL}$. A análise química imediata foi realizada para a quantificação dos teores de materiais voláteis, cinzas e, por diferença, carbono fixo, seguindo as diretrizes da norma ASTM D1762-84 (American society for testing materials, 2007). Os componentes elementares (C, H, N, S e O, obtido por diferença) foram quantificados em um analisador universal de macro (Elementar, Vario Micro Cube), utilizando como gases de arraste e ignição, o hélio e o oxigênio, respectivamente. O ensaio seguiu a metodologia apresentada em Protásio et al. (2011). O poder calorífico superior (PCS) foi determinado em um calorímetro digital (IKA, C-200) de acordo com os procedimentos descritos na ASTM E711-87 (American society for testing materials, 2004). O poder calorífico inferior (PCI) (base seca) e o poder calorífico líquido (PCL) foram estimados com as equações 1 e 2 de acordo com Faria et al. (2016). A densidade energética foi obtida pelo produto entre a densidade e o poder calorífico líquido. 


$$
P C I=P C S-\left(600 \times \frac{9 H}{100}\right)
$$

Onde: $\mathrm{PCI}$ = Poder calorífico inferior; $\mathrm{PCS}=$ Poder calorífico superior; $\mathrm{H}=$ Teor de hidrogênio.

$$
P C L=P C I \times\left[\left(\frac{100-U b u}{100}\right)-(6 \times U b u)\right]
$$

Onde: $\mathrm{PCL}=$ Poder calorífico líquido; $\mathrm{PCI}=$ Poder calorífico inferior; Ubu= Umidade na base úmida.

\subsection{Análise estatística}

Os dados foram expressos como os valores médios de três análises. Após a análise de variância, as médias, quando significativas, foram comparadas pelo teste de Tukey a um nível de 5\% de significância, considerando um delineamento inteiramente casualizado (DIC). As análises foram realizadas no software Sisvar, versão (5.6) (Ferreira, 2019).

\section{Resultados e Discussão}

\subsection{Caracterização dos resíduos}

Os valores médios e coeficiente variação das propriedades físicas e energéticas dos resíduos de café e finos de carvão vegetal estão apresentados na Tabela 2. A análise química imediata (material volátil, carbono fixo e teor de cinzas) é de suma importância para a avaliação energética de um material, pois são variáveis que influenciam no poder calorífico, sendo que, geralmente quanto maior o teor de carbono fixo e menor os teores de materiais voláteis e cinzas, maior é o potencial calorífico do combustível, isto porque a queima ocorre de forma mais lenta e eficiente (Loureiro et al., 2021). Esse comportamento foi observado em parte nos resíduos avaliados, onde os finos de carvão vegetal tiveram o melhor desempenho energético em relação à biomassa residual do café, devido a maior quantidade de carbono fixo e menor teor de materiais voláteis, apesar do seu elevado teor de cinzas. Esses resultados sugerem os finos de carvão vegetal como uma fonte potencial de carbono fixo para ser incorporada em biocombustíveis sólidos, visando à melhoria das propriedades energéticas.

Tabela 2. Teor de umidade, química imediata e poder calorífico de biomassa residual do café e finos de carvão vegetal.

\begin{tabular}{lccc}
\multicolumn{1}{c}{ Análise } & Resíduo do café & Finos de carvão vegetal & CVe(\%) \\
\hline Umidade na base úmida (\%) & $9,78^{\mathrm{a}}$ & $6,26^{\mathrm{b}}$ & 4,11 \\
Umidade na base seca (\%) & $10,85^{\mathrm{a}}$ & $6,68^{\mathrm{b}}$ & 4,39 \\
Teor de voláteis (\%) & $73,59^{\mathrm{a}}$ & $5,42^{\mathrm{b}}$ & 0,86 \\
Carbono fixo (\%) & $20,30^{\mathrm{b}}$ & $53,62^{\mathrm{a}}$ & 0,97 \\
Teor de cinzas (\%) & $6,11^{\mathrm{b}}$ & $20,95^{\mathrm{a}}$ & 4,09 \\
Poder calorífico superior (MJ.Kg $\left.{ }^{-1}\right)$ & $18,40^{\mathrm{b}}$ & $21,43^{\mathrm{a}}$ & 1,20 \\
Poder calorífico líquido (MJ.Kg & $15,21^{\mathrm{b}}$ & $19,49^{\mathrm{a}}$ & 1,27 \\
Carbono (\%) & $45,71^{\mathrm{a}}$ & $46,53^{\mathrm{a}}$ & 2,21 \\
Oxigênio (\%) & $45,32^{\mathrm{b}}$ & $50,05^{\mathrm{a}}$ & 2,39 \\
Nitrogênio (\%) & $3,05^{\mathrm{a}}$ & $1,22^{\mathrm{b}}$ & 3,15 \\
Hidrogênio (\%) & $5,62^{\mathrm{a}}$ & $2,10^{\mathrm{b}}$ & 2,05 \\
Enxofre (\%) & $0,31^{\mathrm{a}}$ & $0,09^{\mathrm{b}}$ & 23,47 \\
\hline
\end{tabular}

$\mathrm{CVe}=$ coeficiente de variação experimental. Médias na linha seguidas da mesma letra não diferem entre si pelo teste de Tukey a 5\% de significância. Fonte: Autores. 


\subsection{Qualidade das misturas residuais para uso energético}

A inclusão de finos de carvão vegetal promoveu a redução significativa da umidade da biomassa lignocelulósica de café (Figura 2AB). O tratamento com 30\% de finos de carvão apresentou uma diminuição da umidade na base úmida e seca de aproximadamente 11 e $12 \%$, respectivamente. Isso ocorreu provavelmente devido a baixa umidade dos finos de carvão (6,26 e 6,68 \%) em relação à biomassa de café $(9,78$ e 10,85 \%), Tabela 2 . A umidade é um parâmetro muito importante quando se avalia biomassa vegetal para fins energéticos, pois a água atua negativamente no valor calórico, devido à perda energética inicial necessária para secar o material.

Figura 2. Umidade base úmida (A) e base seca (B) de resíduos de café e suas misturas com finos de carvão vegetal.

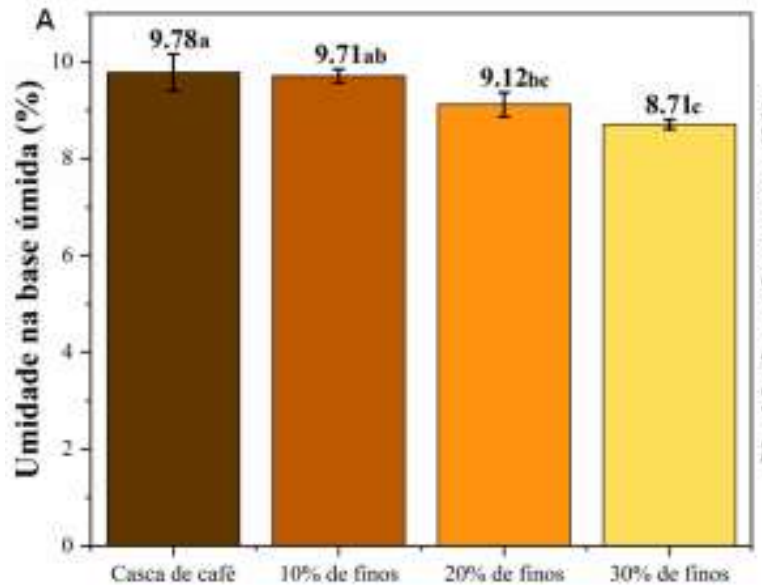

Tratamento

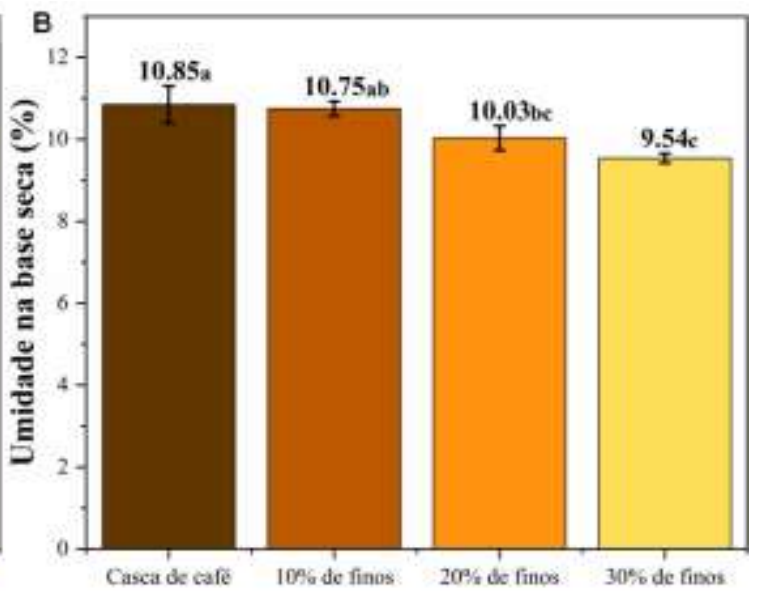

Tratamento

Em que: Valores médios seguidos pela mesma letra para cada tratamento não diferem estatisticamente pelo teste de Tukey a $5 \%$ de significância. Fonte: Autores.

Na Figura 3, constam os valores médios de poder calorífico do resíduo de café e de suas misturas com finos de carvão vegetal. O poder calorífico é uma importante propriedade na determinação do potencial energético de uma biomassa vegetal, pois representa a quantidade de energia liberada na combustão completa de uma unidade de massa do combustível (Pinto et al., 2021). O tratamento com $30 \%$ de finos de carvão vegetal apresentou a melhor qualidade, atingindo um poder calorífico superior de 19,44 $\mathrm{MJ} \mathrm{Kg}^{-1}$ e poder calorífico líquido de 16,75 $\mathrm{MJ} \mathrm{Kg}^{-1}$ (Figura 3AB), um aumento energético de $6 \mathrm{e}$ $10 \%$, respectivamente, em relação ao tratamento com $100 \%$ de biomassa residual de café.

Figura 3. Poder calorífico superior (A) e líquido (B) de resíduos de café e suas misturas com finos de carvão vegetal.

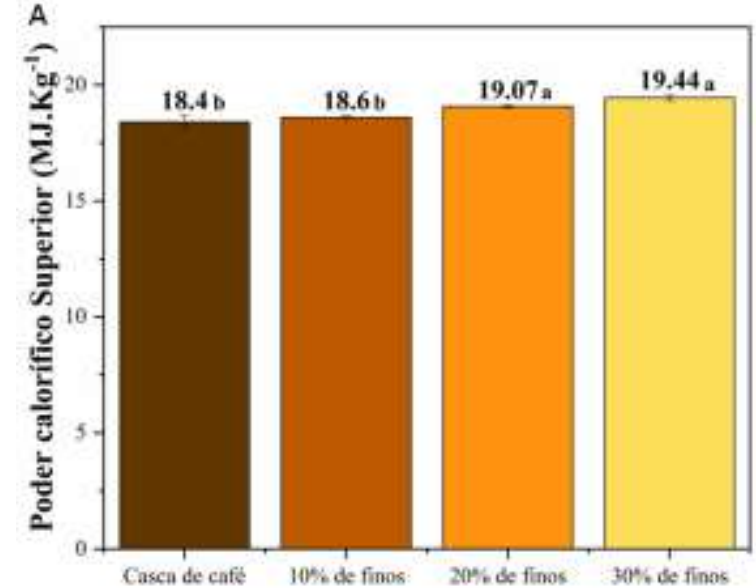

Tratamento

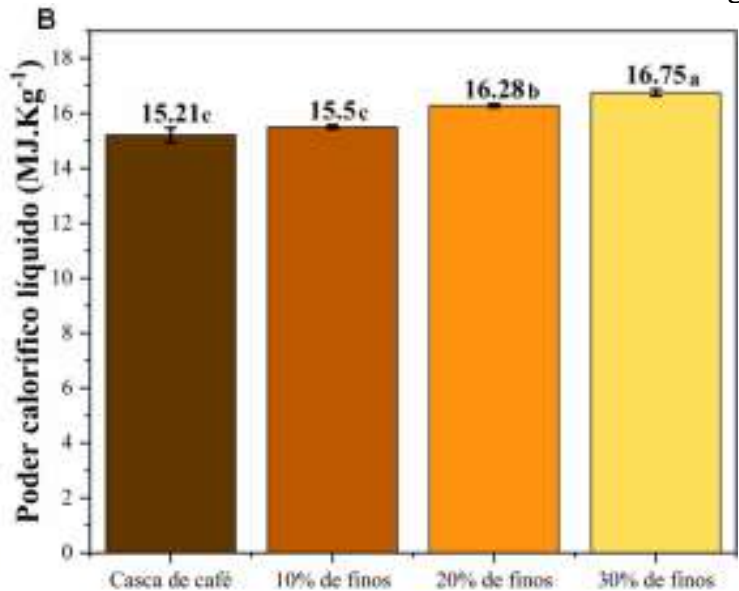

Tratamento

Em que: Valores médios seguidos pela mesma letra para cada tratamento não diferem estatisticamente pelo teste de Tukey a $5 \%$ de significância. Fonte: Autores. 
Em comparação com outros estudos, os resultados de poder calorífico superior das misturas residuais são superiores ao da biomassa residual de café $\left(18,44 \mathrm{MJ} \mathrm{Kg}^{-1}\right)$ e dos briquetes produzidos com os resíduos do processamento dos grãos de café $\left(18,00 \mathrm{MJ} \mathrm{Kg}^{-1}\right)$ relatados por Protásio et al. (2011; 2012). O poder calorífico líquido dos tratamentos com 20 e $30 \%$ de finos de carvão vegetal superaram também os valores encontrados por Faria et al. (2016) para pellets produzidos com casca de café $\left(15,84 \mathrm{MJ} \mathrm{Kg}^{-1}\right)$ e com misturas de casca e película prateada de café $\left(16,19 \mathrm{MJ} \mathrm{Kg}^{-1}\right)$. Em relação a outros tipos de biomassas lignocelulósicas, o PCS médio das misturas residuais (Figura 3A) é próximo ao encontrado para o epicarpo de macaúba - Acrocomia aculeata (20,36 $\mathrm{MJ} \mathrm{Kg}^{-1}$ ) e madeira de Pinus sp. (21,30 $\mathrm{MJ} \mathrm{Kg}^{-1}$ ) (Costa et al., 2019), e maior do que aqueles quantificados para diferentes cultivares de aveia - Avena spp. (17,9 $\mathrm{MJ} \mathrm{Kg}^{-1}$ ) (Pinto et al., 2021) e cascas dos frutos de Munguba - Pachira aquatica (16,24 $\mathrm{MJ} \mathrm{Kg}^{-1}$ ) (Correia et al., 2022). Essa análise comparativa torna evidente a qualidade dos finos de carvão vegetal na agregação de valor energético em resíduos do processamento do café (Figura 3AB).

A densidade a granel teve um aumento significativo com a adição de finos de carvão vegetal, passando de 167,70 para 201,77 Kg.m ${ }^{-3}$ com 30\% de finos (Figura 4A). Os tratamentos com 10, 20 e 30\% de finos de carvão não apresentaram diferença estatística nos valores de densidade a granel. Segundo Costa et al. (2019), valores baixo de densidade a granel (80$150 \mathrm{~kg} \mathrm{~m}^{-3}$ ) são um dos fatores que limitam a utilização de resíduos agrícolas em produtos bioenergéticos, devido sua influência na densidade energética e nos custos de transporte. Neste contexto, os resíduos oriundos do beneficiamento do café possuem potencial para uso energético, principalmente quando agregados com os finos de carvão vegetal, haja vista que promoveram o aumento da densidade energética (Figura 4B), sugerindo o uso potencial dessas misturas residuais para biocombustíveis compactados.

Figura 4. Densidade a granel (A) e densidade energética (B) de resíduos de café e suas misturas com finos de carvão vegetal.

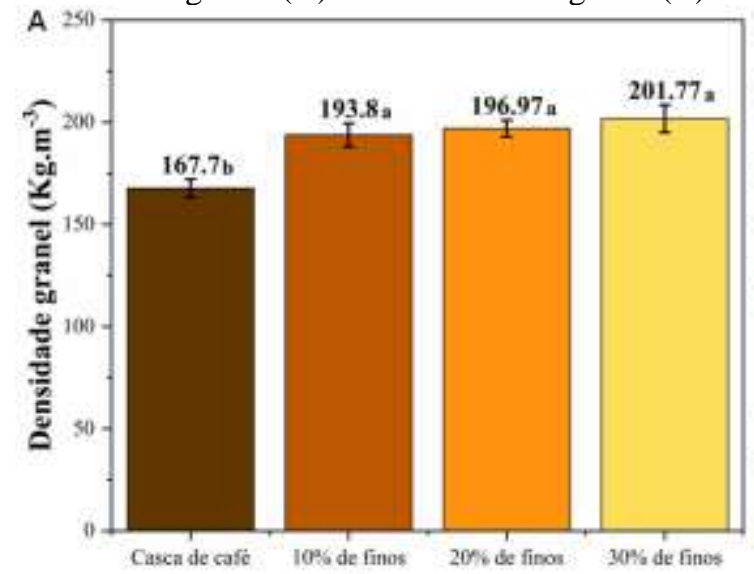

Tratamento

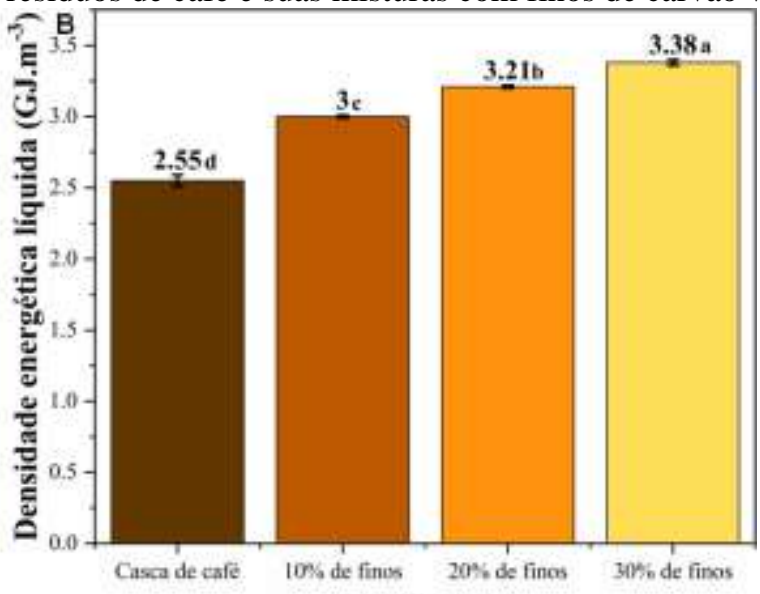

Tratamento

Em que: Valores médios seguidos pela mesma letra para cada tratamento não diferem estatisticamente pelo teste de Tukey a $5 \%$ de significância. Fonte: Autores.

\section{Conclusão}

Os resíduos do processamento dos grãos de café e os finos de carvão vegetal possuem uma composição físicoquímica que justificam seu aproveitamento para a geração de energia térmica, podendo ser uma alternativa viável economicamente e ambientalmente diante da sua disponibilidade. Os finos de carvão vegetal promoveram o incremento energético da biomassa residual do café. Os tratamentos com 20 e 30\% de finos de carvão vegetal se mostraram mais eficientes, devido a menor umidade e o maior poder calorífico e densidade energética. Portanto, recomenda-se a mistura de pelo menos $20 \%$ de finos de carvão vegetal para potencializar o desempenho energético de resíduos oriundos da produção de café. Além do mais, vale ressaltar que as misturas residuais avaliadas não foram densificadas, assim, acreditamos que o 
processo de compactação da biomassa possa aumentar a densidade energética e promover mais ganho energético. Desta forma, sugerimos que as misturadas estudadas sejam avaliadas após a compactação na forma de pellets e/ou briquetes.

\section{Referências}

Angeloni, G., Guerrini, L., Masella, P., Innocenti, M., Bellumori, M., \& Parenti, A. (2019). Characterization and comparison of cold brew and cold drip coffee extraction methods. Journal of the Science of Food and Agriculture, 99(1), 391-399. https://doi.org/10.1002/jsfa.9200

American Society For Testing Materials. (2004). ASTM E711-87 : Standard Test Method for Gross Calorific Value of Refuse-Derived Fuel by the Bomb Calorimeter. Philadelphia: ASTM International, $8 \mathrm{p}$.

American Society For Testing Materials. (2007). ASTM D 1762- 84 : Standard method for chemical analyses of wood charcoal. Philadelphia: ASTM International, $2 \mathrm{p}$.

Anwar, Z., Gulfraz, M., \& Irshad, M. (2014). Agro-Industrial Lignocellulosic Biomass a Key to Unlock the Future Bio-Energy: A Brief Review.” Journal of Radiation Research and Applied Sciences, 7(2): 163-73. http://dx.doi.org/10.1016/j.jrras.2014.02.003.

Awan, F. U. R., Keshavarz, A., Akhondzadeh, H., et al. (2020). Stable Dispersion of Coal Fines during Hydraulic Fracturing Flowback in Coal Seam Gas Reservoirs-An Experimental Study. Energy \& Fuels, 34(5), 5566-5577. http://dx.doi.org/10.1021/acs.energyfuels.0c00045

Bajpai, P. (2016). Structure of Lignocellulosic Biomass. 7-12. https://doi.org/10.1007/978-981-10-0687-6_2

Correia, L. A. da S., Silva, J. E. da, Calixto, G. Q., Melo, D. M. de A., \& Braga, R. M. (2022). Pachira aquatica fruits shells valorization: Renewables phenolics through analytical pyrolysis study (Py-GC/MS). Ciência Rural, 52(2), e20210068. https://doi.org/10.1590/0103-8478cr20210068

Costa, S. E. de L., Santos, R. C. dos, Castro, R. V. O., Castro, A. F. N. M., Magalhães, M. A. de, Carneiro, A. de C. O., Santos, C. P. de S., Gomes, I. R. F., \& Rocha, S. M. G. (2019). Briquettes quality produced with the macauba epicarp (Acrocomia aculeata) and Pinus sp. wood. Revista Árvore, 43(5), e430501. https://doi.org/10.1590/1806-90882019000500001

Cubero-Abarca, R., Moya, R., Valaret, J., \& Tomazello Filho, M. (2014). Use of coffee (Coffea arabica) pulp for the production of briquettes and pellets for heat generation. Ciência e Agrotecnologia, 38(5), 461-470. https://doi.org/10.1590/s1413-70542014000500005

DEUTSCHES INSTITUT FÜR NORMUNG. (2010). DIN EN 14774-1 : Determination of moisture content - Oven dry method - Part 1: Total moisture Reference method. Berlin: CEN, 10 p. 14774

Demirbas, A. (2002). Relationships between heating value and lignin, moisture, ash and extractive contents of biomass fuels. Energy Exploration \& Exploitation, 20(1):105-111. https://doi.org/10.1260/014459802760170420

Dias Júnior, A.F., Suuchi, M.A., Sant'Anna Neto, A. et al. (2021). Blends of charcoal fines and wood improve the combustibility and quality of the solid biofuels. Bioenerg. Res. 14, 344-354. https://doi.org/10.1007/s12155-020-10179-8

Faria, W., De, T., Protásio, T., Trugilho, P., Luísa, B., Pereira, C., Carneiro, A., Carneiro, O., Andrade, C., Benedito, J., \& Junior, G. (2016). Transformação dos resíduos lignocelulósicos da cafeiculTura em Pellets para geração de energia Térmica. Coffee Science, 11, $137-147$.

Ferreira, D. F. (2019). A computer analysis system to fixed effects split plot type designs. Rev. Bras. Biometria, 37, 529-535. https://doi.org/10.28951/rbb. v37i4.450.

Jeguirim, M., Limousy, L., \& Fossard, E. (2016). Characterization of coffee residues pellets and their performance in a residential combustor. International Journal of Green Energy, 13(6), 608-615. https://doi.org/10.1080/15435075.2014.888664

Loureiro, B. A., Assis, M. R., Melo, I. C. N. A., Oliveira, A. F. C. F., \& Trugilho, P. F. (2021). Rendimento gravimétrico da carbonização e caracterização qualitativa do carvão vegetal em clones de híbridos de Corymbia spp para uso industrial. Ciência Florestal, 31(1), 214-232. https://doi.org/10.5902/1980509836120

Nosek, R., Tun, M. M., \& Juchelkova, D. (2020). Energy utilization of spent coffee grounds in the form of pellets. Energies, 13(5), 1-8. https://doi.org/10.3390/en13051235

Okolie, J. A., Nanda, S., Dalai, A. K., \& Kozinski, J. A. (2021). Chemistry and Specialty Industrial Applications of Lignocellulosic Biomass. Waste and Biomass Valorization, 12(5), 2145-2169. https://doi.org/10.1007/s12649-020-01123-0

Pinto, C. W., Barth, G., Molin, R., Silva, D. A. D., \& Pauletti, V. (2021). Characterization of oat biomass for energy production. Revista Caatinga, 34(3), 537-547. https://doi.org/10.1590/1983-21252021v34n305rc

Protásio, T. de P., Alves, I. C. N., Trugilho, P. F., Silva, V. O., \& Baliza, A. E. R. (2011). Compactação de biomassa vegetal visando à produção de biocombustíveis sólidos. Pesquisa Florestal Brasileira, 31(68), 273-283. https://doi.org/10.4336/2011.pfb.31.68.273

Protásio, T. de P., Bufalino, L., Mendes, R. F., Ribeiro, M. X., Trugilho, P. F., \& Leite, E. R. da S. (2012). Torrefação e carbonização de briquetes de resíduos do processamento dos grãos de café. Revista Brasileira de Engenharia Agrícola e Ambiental, 16(11), 1252-1258. https://doi.org/10.1590/S141543662012001100015

Rana, R., Nanda, S., Kozinski, J. A., \& Dalai, A. K. (2018). Investigating the applicability of Athabasca bitumen as a feedstock for hydrogen production through catalytic supercritical water gasification. Journal of Environmental Chemical Engineering, 6(1), 182-189. https://doi.org/10.1016/j.jece.2017.11.036 
Setter, C., Borges, F. A., Cardoso, C. R., Mendes, R. F., \& Oliveira, T. J. P. (2020). Energy quality of pellets produced from coffee residue: Characterization of the products obtained via slow pyrolysis. Industrial Crops and Products, 154, 112731. https://doi.org/10.1016/j.indcrop.2020.112731

Shan, F., Lin, Q., Zhou, K., Wu, Y., Fu, W., Zhang, P., Song, L., Shao, C., \& Yi, B. (2017). An experimental study of ignition and combustion of single biomass pellets in air and oxy-fuel. Fuel, 188, 277-284. https://doi.org/10.1016/j.fuel.2016.09.069

Solarte-Toro, J. C., Chacón-Pérez, Y., \& Cardona-Alzate, C. A. (2018). Evaluation of biogas and syngas as energy vectors for heat and power generation using lignocellulosic biomass as raw material. Electronic Journal of Biotechnology, 33, 52-62. https://doi.org/10.1016/j.ejbt.2018.03.005

Souza, H. J. P. L., Arantes, M. D. C., Vidaurre, G. B., Andrade, C. R., Carneiro, A. de C. O., de Souza, D. P. L., \& Protásio, T. de P. (2020). Pelletization of eucalyptus wood and coffee growing wastes: Strategies for biomass valorization and sustainable bioenergy production. Renewable Energy, 149, 128-140. https://doi.org/10.1016/j.renene.2019.12.015

Tawalbeh, M., Al-Othman, A., Salamah, T., Alkasrawi, M., Martis, R., \& El-Rub, Z. A. (2021). A critical review on metal-based catalysts used in the pyrolysis of lignocellulosic biomass materials. Journal of Environmental Management, 299, 113597. https://doi.org/10.1016/j.jenvman.2021.113597

Tian, S. Q., Zhao, R. Y., \& Chen, Z. C. (2018). Review of the pretreatment and bioconversion of lignocellulosic biomass from wheat straw materials. Renewable and Sustainable Energy Reviews, 91, 483-489. https://doi.org/10.1016/j.rser.2018.03.113 